"(C) ( 2010 IEEE. Personal use of this material is permitted. Permission from IEEE must be obtained for all other uses, in any current or future media, including reprinting/republishing this material for advertising or promotional purposes, creating new collective works, for resale or redistribution to servers or lists, or reuse of any copyrighted component of this work in other works."

The definitive version of this article is available at http://ieeexplore.ieee.org

Published as: Castro, H.; Alves, A.P.; Serrao, C.; Caraway, B., "A New Paradigm for Content Producers," MultiMedia, IEEE, vol.17, no.2, pp.90-93, April-June 2010 DOI: http://dx.doi.org/10.1109/MMUL.2010.26 


\section{A New Paradigm for Content Producers}

Helder Castro and

Artur P. Alves INESC Porto

Carlos Serrão ISCTE-IUL, Portugal

Brett Caraway University of

Texas
$\mathbf{T}$ he production, distribution, and consumption of information goods have endured numerous challenges over the years. Most recently, the Internet and digital consumer technologies have severely disrupted established intellectual-property regimes, enabling the near costless reproduction and distribution of information commodities. In addition, sophisticated tools have enabled new collaborative spaces (such as blogs, social websites, and so forth) for media production and distribution, posing new challenges to traditional creatorproducer-consumer paradigms.

This article analyzes the present situation's main technological characteristics, its economic implications, and the industry's response-and outlines a possible solution to the problems.

\section{Present scenario}

The Internet is quickly becoming the main channel for distributing information goods. ${ }^{1}$ It greatly reduces or eliminates the costs of conveniently and efficiently searching for, retrieving, and distributing these goods, and potentially signals an end to the era of mass physical distribution. The proliferation of Internet technologies both puts pressure on information producers to adopt online distribution systems as well as invalidates their previously solid business models. In the Internet era, the entities that produce and distribute information goods, such as music producers, film studios, and so forth, have found themselves in an uncomfortable position.

In the past, what secured the profits on intellectual property was the scarcity that was intrinsic to the materiality of the distribution structure. As that materiality is eroded, so is the control producers have over content use. According to the Recording Industry Association of America, the music industry loses about $\$ 12.5$ billion per year from all types of piracy activities. ${ }^{2}$ The accuracy of these numbers is disputed, ${ }^{3}$ but it's still plausible that considerable revenue losses have occurred as a result of the rapid increase in the free exchange of copyrighted content over the Internet.

The current business methods of these content producers, along with their long-term sustainability, are therefore threatened. It seems clear, then, that it has become necessary to replace the hitherto reliable value chain in which information commodities have been traded. The production and distribution of information commodities can no longer be approached in the same way as the production and distribution of physically bound information commodities.

Despite the new realities of Internet-era production and consumption, the creatorproducer industry has reacted very conservatively to these issues, attempting to preserve the operational status quo by artificially maintaining the scarcity of its information products. In this context, producers have employed two main courses of action. The first was legislative and punitive action against accused copyright infringers. The second was technological measures known as digital rights management (DRM). The industry response, in other words, has been to resist or attempt to reverse social 
and technological development with respect to the free flow of information.

\section{Industry response results}

The industry's conservative response has produced little or no results. Efforts aimed at tightening intellectual property laws were relatively successful in some countries (for example, the Digital Millennium Copyright Act in the US), and the frequent legal actions against alleged copyright infringers have led to some legal success. Still, such measures have had little impact in terms of deterring content sharing and have created considerable dissatisfaction from the consumer and small-creator and producer communities, causing costly damages to the industry's public reputation. ${ }^{4}$

In addition, the end-user circumvention and rejection of DRM has been a near constant, with digital piracy only increasing despite the prevalence of new and increasingly elaborate DRM strategies. ${ }^{5}$ The approach adopted by producers has thus failed, with control over information commodities now rapidly decreasing. Furthermore, online content sharing has persevered to the point that many Web-based retail outlets have abandoned DRM altogether in favor of unprotected content so as to offer a product that is not hampered by a lack of usability (which inevitably comes with DRM) in its competition with pirate content. ${ }^{6,7}$

The failure of DRM is the result of several weaknesses, especially its intrinsic vulnerability. For example, users must inevitably be given access to each system's secret contentencoding key. As a result, all mainstream DRM systems (such as Apple's FairPlay, Microsoft's Windows Media DRM, and so forth) have been circumvented. In addition, DRM imposes usage restrictions that devalue the content and push consumers away. Finally, DRM isolates users because all unique DRM systems lack interoperability, ${ }^{8}$ which means users are confined to the vendor's software or hardware.

DRM's failure means that the producers must still bear the fixed costs of information production, but their monetization schemes are losing efficiency. Their privileged foundation is rapidly eroding and they are evermore competing in an abundance environment despite the fact that their operation mode depends on scarcity. Clearly, the countermeasures employed by the producers have backfired, only earning producers widespread animosity from the very people they are trying to win as customers.

These countermeasures have spurred the development of even more decentralized content-sharing systems (such as Gnutella and BitTorrent), which are more resilient to legal and technical counteraction. In Michael Porter's terminology, ${ }^{9}$ these measures merely built barriers to the entry of new legal competitors. In light of these new developments, producers must employ a new approach that, instead of fruitlessly trying to curb sharing, embraces it, accepting that information scarcity is a thing of the past.

It is our belief that future DRM tools must refocus from an access-restriction approach to one which employs a much more flexible kind of rights management so that content access is maximized to support abundance-based business models. Such solutions are not yet available in present DRM technologies. We believe a paradigm shift is necessary in the way the content industry conducts its business, in the way intellectual rights are viewed and managed, and in the way the creators are rewarded. Our solution is to embrace this paradigm shift at the business-model level.

\section{New paradigm}

Technical and economic changes created by the Internet and related technologies are revolutionizing the way information is generated, distributed, and consumed. That revolution must be economically, culturally, and socially embraced through a change of operational paradigm. The valuable resource to be harnessed no longer is the information commodity; instead, it is the user's attention. The producers must work to maximize access to their content and not restrict it. They should perform an immaterial open delivery of their goods that facilitates consumption and exchange. Liberated from the need to control content access, the producers will then be able to harness the Internet's potential for distribution and reproduction.

In this paradigm, content misuse thus amounts only to corruption, adulteration, or wrongful creation attribution. Impeding such misuse should now be the main concern of the producers. In the past, the existence of physical shelf availability for a product required high sales expectation, creating a blockbuster culture that devalued all others goods. 
The immateriality intrinsic to the paradigm we propose gives the producers unlimited shelf space and grants new significance to retail goods. In this scenario, producers focus on selling less of more, and on extracting smaller gains from a greater variety of goods. ${ }^{10}$

Such gains should be made though indirect means, by capitalizing on user attention instead of direct sales. Consumer loyalty is thus paramount to producer operation in the Internet era. It can be secured no longer through the maintenance of a gatekeeper position. It must be achieved by inscribing the contentaccessing activities in a captivating virtual environment of rich social interaction between consumers and original creators (artists). The reward for content creators should be directly dependent on the user community's will, a factor that will foster the creation of emotional and socioeconomic bonds between users and artists.

In this paradigm, consumers will realize that their voluntary contribution is necessary for the continued satisfaction of their intellectual wants. As a result, a culture of voluntary funding and interdependency will emerge. The producer operation can then be sustained by exploiting the mentioned interaction environments, such as by taxing user donations to creators or selling user attention to advertisers. The producers must therefore refocus their efforts on maintaining a virtual social space in which the interaction between consumers, artists, and information goods can take place freely, on fostering the production of quality information goods, on facilitating the distribution of information goods through secondary channels, and on guaranteeing the authenticity and integrity of information goods.

\section{New business model}

A business model for Internet-era producers must enable the free retrieval and consumption of information products while ensuring that such activities are primarily taking place in a producer-managed virtual space. The business model must exploit all possible content niches and sources, extract value from the monetary transactions occurring between consumers and creators within that space, and exploit the Internet's capacity to eliminate reproduction and distribution costs.

We believe that in a donation-based business model, information items will be voluntarily delivered by artists expecting to be monetarily rewarded. Consumers will freely access such items and reward the creators they deem meritorious through donations performed in an intraspace currency that users acquire with real currency or by selling their attention (such as through viewing advertisements).

In an infrastructure created to support this business model, the producer mediates all donations, retaining a fraction of the transacted amounts. Such donations may be a posteriori, in which case consumers reward artists for products already delivered, or a priori, in which case more reputable creators specify that a minimum collective donation must be reached before a specific product is released for public consumption.

A donation-based business model could work with the delivery of any kind of information product, such as video, audio, text, and so forth. This model would replace only the product's material distribution (such as a DVD hard copy) with an immaterial open-access distribution, without regard to other parts of the value chain (such as cinematic presentation, concerts, and so forth).

From our perspective, a donation-based business model would not represent a final discussion in terms of Internet-era models. It would be one possible model among potentially many more. Furthermore, in the context of a donationbased business model, DRM technologies could easily be repurposed to focus on ensuring the authenticity and the validity of information products as well as on content-usage monitoring.

\section{Conclusions}

Reversing the ongoing developments in information content production, reproduction, and distribution would demand the implementation of draconian legal and technical measures, which would have a profoundly impoverishing and undemocratic impact on the way we access and exchange information. Furthermore, the present emergence of social practices based on the free flow of information is a continuation of a longstanding trend that is shifting the socioeconomic support for this field of human activity from voluntary upstream support (patronage) to voluntary downstream support (user donation).

To persevere, those producing information goods should not fight these changes but embrace them by altering their operational paradigm. 
The controlling gatekeeper role occupied for centuries by the producers should be seen as only a temporary one that enables the transitioning of this field of activity from the preindustrial patronage era to the informationindustry era. The role of the producers in this era should now be to maximize content distribution and access, to support captivating virtual social spaces, to promote artist and consumer bonding, and to foster voluntary consumer donations.

MM

\section{References}

1. Organization for Economic Co-Operation and Development, OECD Information Technology Outlook, 2006; http://www.oecd.org/document/1/0,3343,en_ 2649_34223_37486858_1_1_1_1,00.html.

2. Recording Industry Association of America, "Piracy: Online and On The Street," 2008; http://www. riaa.com/physicalpiracy.php.

3. K. Fisher, "The Problem with MPAA's Shocking Piracy Numbers," Ars Technica, 2006; http:// arstechnica.com/old/content/2006/05/6761.ars.

4. N. Anderson, "Five Years of Failure: EFF Says RIAA Must Embrace New Model," Ars Technica, 2008; http://arstechnica.com/tech-policy/news/2008/ 10/five-years-of-failure-eff-says-riaa-must-embracenew-model.ars.
5. T. Karagiannis et al., "Is P2P Dying or Just Hiding?" Proc. IEEE Globecom 2004-Global Internet and Next Generation Networks, vol. 3, 2004, pp. 1532-1538.

6. Z. Mutter, "Universal Offers DRM-free Music," PC Advisor, 2007; http://www.pcadvisor.co.uk/news/ index.cfm?newsid=8675.

7. EMI Group, "EMI Music Launches DRM-Free Superior Sound Quality Downloads Across Its Entire Digital Repertoire," 2007; http://www. emigroup.com/Press/2007/press18.htm.

8. R. Koenen et al., "The Long March to Interoperable Digital Rights Management," Proc. IEEE, vol. 92, no. 6, 2004, pp. 883-897.

9. M. Porter, Competitive Advantage-Creating and Sustaining Superior Performance, Free Press, 2004.

10. C. Anderson, The Long Tail: Why the Future of Business is Selling Less of More, Hyperion, 2006.

Contact author Helder Castro at hcastro@ inescporto.pt.

Contact editor John R. Smith at jsmith@ us.ibm.com.

cn Selected CS articles and columns are also available for free at http://ComputingNow. computer.org. 